Research Article

\title{
Interfacial and Micellization Behavior of Cetyltrimethylammonium Bromide (CTAB) in Water and Methanol-Water Mixture at 298.15 to 323.15 K
}

\author{
Sujit Kumar Shah (i) and Ajaya Bhattarai $(D)$ \\ Department of Chemistry, M. M. A. M. C., Tribhuvan University, Biratnagar, Nepal \\ Correspondence should be addressed to Ajaya Bhattarai; bkajaya@yahoo.com
}

Received 3 March 2020; Revised 28 April 2020; Accepted 23 May 2020; Published 29 June 2020

Academic Editor: Franck Rabilloud

Copyright (C) 2020 Sujit Kumar Shah and Ajaya Bhattarai. This is an open access article distributed under the Creative Commons Attribution License, which permits unrestricted use, distribution, and reproduction in any medium, provided the original work is properly cited.

\begin{abstract}
The micellization behavior of cetyltrimethylammonium bromide (CTAB) in water, $0.1,0.2,0.3$, and 0.4 volume fractions of methanol at $298.15,308.15,318.15$, and $323.15 \mathrm{~K}$ were investigated by surface tension measurements. The effect of methanol on values of critical micelle concentration $(\mathrm{cmc})$, free energies of micellization $\left(\Delta G_{\mathrm{m}}^{\mathrm{o}}\right)$, and surface properties viz. maximum surface excess concentration $\left(\Gamma_{\max }\right)$, area occupied by per surfactant molecule $\left(A_{\min }\right)$, surface pressure $\left(\pi_{\mathrm{cmc}}\right)$, solution surface tension $\left(\gamma_{\mathrm{cmc}}\right)$, solvent surface tension $\left(\gamma_{\mathrm{o}}\right)$, free energies of adsorption $\left(\Delta G_{\mathrm{ads}}^{\mathrm{o}}\right)$, the efficiency of adsorption $\left(p C_{20}\right)$, effective Gibbs free energy $\left(\Delta G_{\text {eff }}^{o}\right)$, and free energy of surface at equilibrium $\left(G_{\min }\right)$ were investigated using surface tension values. Other parameters such as the packing parameter $(P)$, aggregation number $(N)$, concentration of surfactant in the bulk phase $\left(C_{20}\right)$, relation between $A_{\min }$ and $\pi_{\mathrm{cmc}}$, and correlation of slopes $(\mathrm{d} c / \mathrm{d} \log C), \gamma_{\mathrm{o}} / \mathcal{c}_{\mathrm{cmc}}, \Gamma / \Gamma_{\max }, \mathrm{cmc} / C_{20}, \Delta G_{\mathrm{ads}}^{\mathrm{o}} / \Delta G_{\mathrm{m}}^{o}$, and cmc/p $C_{20}$ with the volume fraction of methanol are calculated and discussed in the light of the experiment done.
\end{abstract}

\section{Introduction}

Surfactants are a group of organic compounds with a hydrophilic head and a hydrophobic tail (hence known as amphiphiles) due to which they reduce surface tension and are also known as surface-active agents. They are used in almost all areas of applications because of their unique properties. It is well-known that the head part shows the hydrophobic nature and tail shows hydrophilic nature. As a result, the molecules are oriented on the surface of the water, tail aligning towards the air. This behavior is very common to all classes of surfactants. With the increase in the concentration of surfactant, amphiphiles start aggregating in the bulk to form aggregates. The concentration at which this occurs is known as critical micelle concentration $(\mathrm{cmc})$ [1]. The stability of micelle in solution is due to soft interactions between amphiphile molecules. The main soft interactions acting in the amphiphilic self-assembly are hydrogen bonding, hydrophobic effects, electrostatic interaction, and van der Waals forces. Moreover, such interactions are so flexible that a minor disturbance in the system causes significant changes in the physicochemical and surface properties of the surfactant solution [2]. Some factors that directly affect the physicochemical properties and surface properties of the micellar system are additives, temperature, and solvent composition [3-17].

In this study, we explore the effects of solvent composition and temperature on the surface properties of the surfactants. By adding suitable organic cosolvents such as alcohol, there is a measurable change in the hydrophobicity of the medium, which eventually reforms the surface and physicochemical properties of the surfactants [18-22]. By increasing the temperature of the surfactant system, an increase in the cmc can be noticed [20].

Also, we have seen that the surface tension of the surfactant at first decreases with increasing concentration of surfactant and then followed by a sigmoidal curve between surface tension $(\gamma)$ and $\log$ (surfactant). There is a formation 
of the visible break on the sigmoidal curve after which the surface tension remains approximately constant. This concentration is called $\mathrm{cmc}$ [23]. A comprehensive investigation of the effect of methanol on such variations at different temperatures has not found in the literature [24, 25].

Kumar et al. investigated the thermodynamic parameters of SDS and CTAB by measuring the conductivity. They reveal that alcohol plays a vital role in these properties. They concluded that the observed changes in the properties of SDS and CTAB are mainly due to different structural consequences of interactions between molecules of surfactant in mixed solvent media [26]. Bielawska et al. studied the influence of methanol, ethanol, and 1-propanol on CTAB monomeric and aggregated forms. However, detailed investigations of the tensiometric approach are lacking [27]. Zdziennicka and Janczuk measured the surface tension of $\mathrm{CTAB}$ in methanol and ethanol. The concentration of CTAB was in the premicellar range $\left(1 \times 10^{-5} \mathrm{M}\right.$ to $\left.1 \times 10^{-3} \mathrm{M}\right)$. They compared the isotherm of surface tension with those obtained from Szyszkowski and Connors equations. They found that alcohol decreased cationic surfactant adsorption at the water-air interface at low concentration. However, at a concentration close to $\mathrm{cmc}$, the cationic surfactant decreases the adsorption of alcohol considerably [28].

Apart from a conventional surfactant, alcohol as a cosolvent shows substantial variations in micellization in a mixed surfactant system. Isopropanol shows the synergistic effect on mixed micellization of a binary mixture of aminosulfonate amphoteric surfactant with octadecyltrimethyl ammonium bromide in an aqueous medium. The effect is understood by interaction behavior between surfactant molecules in terms of hydrophobic effect [29].

According to the investigation done by Li et al. [22], the presence of short-chain alcohols alters the hydrophobic effect and the electrostatic interaction among amphiphiles and cause the variation in interfacial or micellization behavior. In this study, it was also reported that methanol can fully assist as cosolvent and indicates decent hydrophobicity, which encourages the solubility of surfactant in the aqueous medium. This drew out attention to investigate surface properties of CTAB in the presence of methanol.

In our previous article [30], thermodynamic investigations of DTAB and CTAB have been already published. This article describes the tensiometric approach to CTAB in mixed solvent media. The mixture of water and the organic solvent was termed the mixed solvent media. By changing the solvent composition, the relative permittivity of the media can be varied.

We aim to investigate the aggregation behavior of CTAB in water and the methanol-water mixture containing $0.1,0.2$, 0.3 , and 0.4 volume fraction of methanol at 298.15 to $323.15 \mathrm{~K}$ in detail.

\section{Experimental}

2.1. Materials and Methods. Methanol (99.0\% pure) was purchased from Merck, India. The methanol had a density of $0.7872 \mathrm{~g} / \mathrm{cm}^{3}$ and a coefficient of viscosity of $0.5440 \mathrm{mPa} \cdot \mathrm{s}$ at
298.15 K; these values are also seen in the literature [31]. The cationic surfactant CTAB was purchased from Aldrich Chemical Company, Inc., USA. CTAB was highly pure (>99.0\%) and used after drying for $1 \mathrm{~h}$. The $\mathrm{cmc}$ of CTAB was measured at $298.15 \mathrm{~K}$. The observed $\mathrm{cmc}$ of CTAB by conductivity is $0.9 \mathrm{mM}$, which was matched with the literature [32]. The methanol-water mixtures were prepared up to 0.4 volume fraction of methanol at $298.15 \mathrm{~K}$ by maintaining a constant temperature in a thermostat [18] by using triply distilled water. The concentration range of the CTAB solution was $1 \times 10^{-2} \mathrm{M}$ to $5 \times 10^{-4} \mathrm{M}$.

The specific conductivity measurement of triply distilled water was measured by a digital conductivity meter with a cell constant of $1.002 \mathrm{~cm}^{-1}$ with an uncertainty of $0.01 \% . \mathrm{KCl}$ solution $(0.1 \mathrm{M}$ and $0.01 \mathrm{M})$ was used for calibration of conductivity cell at $298.15 \mathrm{~K}$ [33]. Our purpose of using conductivity meter was to check the conductivity of triply distilled water which was $<0.6 \mu \mathrm{S} / \mathrm{cm}$ at $298.15 \mathrm{~K}$. The uncertainty of the measurements was within $\pm 3.0 \%$.

In the surface tension method, a concentrated solution of $\mathrm{CTAB}$ was prepared in water and mixed solvents of the methanol-water mixture at $298.15 \mathrm{~K}$. The solutions of CTAB were diluted by the internal dilution method with the help of a microsyringe to vary the concentration of CTAB. Each concentration of the CTAB solution was placed in a wide mouth dish with the platinum ring [31]. After each addition, the surface tension of the solution was measured (after thorough mixing and temperature equilibration) using a calibrated Kruss K20S force tensiometer, using the technique of ring detachment [34].

The measurements were triplicated. Measurements were made at 298.15 to $323.15 \mathrm{~K}$ in a thermostated water-bath maintained within $\pm 0.01 \mathrm{~K}$. The uncertainty during the experiments was within $\pm 1 \times 10^{-3} \mathrm{Nm}^{-1}$. To maintain the temperature equilibrium, the tensiometer was connected to a water-flow cryostat.

\section{Results and Discussion}

3.1. Surface Tension Measurements and Surface Properties. The surface tension of the CTAB solutions in water, 0.10 , $0.20,0.30$, and 0.40 volume fractions of methanol-water were plotted against the concentration to get the $\mathrm{cmc}$ values at 298.15, 308.15, 318.15, and $323.15 \mathrm{~K}$ temperatures. The representative Figure 1 shows the variation in the surface tension of CTAB in water, $0.1,0.2,0.3$, and 0.4 volume fractions of methanol at $308.15 \mathrm{~K}$. The surface tension of $\mathrm{CTAB}$ at first decreases with increasing concentration of $\mathrm{CTAB}$, and a sigmoidal curve observed between the surface tension $(\gamma)$ and $\log$ [CTAB] by the distinct break is known as the cmc.

In other words, we can discuss the detailed mechanism of the variation in the surface tension of CTAB with its concentration in the following ways: CTAB adsorbs to the surface only at low concentrations, and then, there is a rapid decrease in the surface tension. When the concentration of CTAB is increased further, the molecules start moving towards bulk instead of getting adsorbed at the surface [35]. 


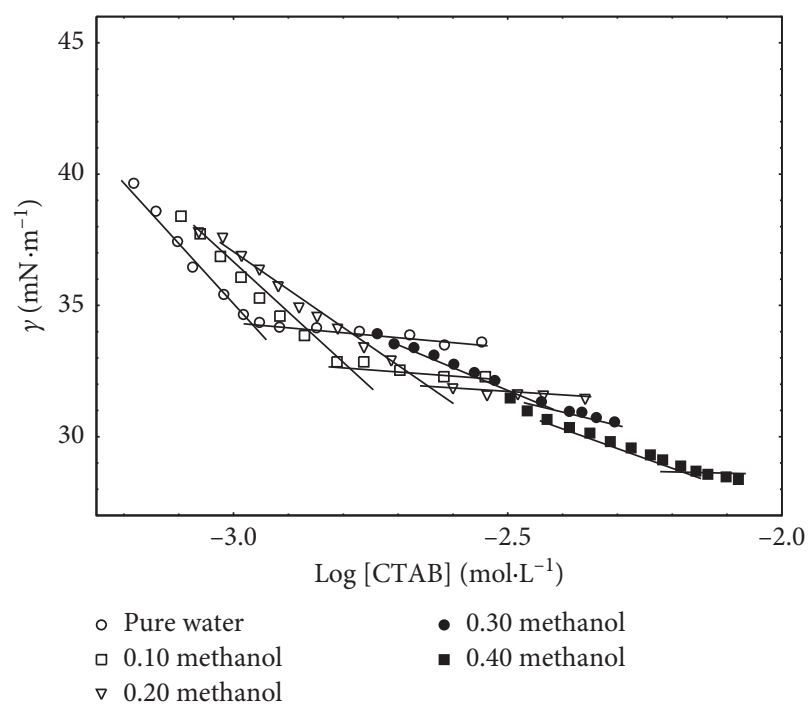

Figure 1: Plot of surface tension versus concentration of CTAB solution at $308.15 \mathrm{~K}$.

From the plot of surface tension with the concentration of $\mathrm{CTAB}$ solution, the following surface properties were calculated [20]. The maximum surface excess concentration at the air/methanol-water interface $\Gamma_{\max }$ was calculated by applying Gibbs isotherm:

$$
\Gamma_{\max }=-\frac{1}{2.303 n R T}\left[\frac{\mathrm{d} \gamma}{\mathrm{d} \log C}\right]_{T, P},
$$

where $\gamma$ denotes the surface tension, $R$ is the gas constant $\left(8.314 \mathrm{~J} \cdot \mathrm{mol}^{-1} \cdot \mathrm{K}^{-1}\right), T$ is the absolute temperature, $C$ is the surfactant concentration, and $(\mathrm{d} \gamma / \mathrm{d} \log C)$ is the slope of the $\gamma$ versus $\log C$ plot taken at $\mathrm{cmc}$. The constant $n$ takes the values 2 for the conventional surfactant where the surfactant ion and the centerline are univalent.

The area occupied per surfactant molecule $A_{\min }$ at the air/methanol-water interface was obtained by

$$
A_{\min }=\frac{1}{N_{A} \Gamma_{\max }}
$$

where $N_{A}$ is Avogadro's number.

The value of the surface pressure at the $\mathrm{cmc}\left(\pi_{\mathrm{cmc}}\right)$ is obtained as

$$
\pi_{\mathrm{cmc}}=\gamma_{\mathrm{o}}-\gamma_{\mathrm{cmc}}
$$

where $\gamma_{\mathrm{o}}$ and $\gamma_{\mathrm{cmc}}$ are the values of surface tension of water and the surfactant solution at the $\mathrm{cmc}$, respectively.

The standard Gibbs free energy of micellization, $\Delta G_{\mathrm{m}}^{\mathrm{o}}$, is calculated from the following relation:

$$
\Delta G_{\mathrm{m}}^{\mathrm{o}}=(2-\alpha) R T \ln X_{\mathrm{cmc}},
$$

where $X_{\mathrm{cmc}}$ is the mole fraction of $\mathrm{cmc}$ and $\alpha$ is the degree of dissociation which is calculated from the ratio of premicellar and postmicellar slopes of the plot of conductivity and concentration.
Since temperature variation is done here, we can try to evaluate free energy by the Van't Hoff method, which is given by the following equation:

$$
\left[\frac{\partial\left\{\Delta G_{\mathrm{m}}^{\mathrm{o}} / T\right\}}{\partial(1 / T)}\right]_{P}=\Delta H_{\mathrm{m}}^{\mathrm{o}}
$$

Equation (5) is fulfilled when the entropy is constant in the studied range of temperature.

The standard free energy interfacial adsorption at the air/ saturated monolayer interface can be evaluated from the following relation:

$$
\Delta G_{\mathrm{ads}}^{\mathrm{o}}=G_{\mathrm{m}}^{\mathrm{o}}-\frac{\pi_{\mathrm{cmc}}}{\Gamma_{\max }}
$$

The surface area of amphiphiles in mixed micelles and micellar growth (spherical-nonspherical) can be used to find out the packing parameters $P$ :

$$
P=\frac{V_{\mathrm{o}}}{A_{\min } l_{c}},
$$

where $V_{\mathrm{o}}$ is the volume of exclusion per monomer in the aggregate, as in Tanford's formula.

$$
V_{\mathrm{o}}=\left[27.4+26.9\left(n_{c}-1\right)\right] 2 \dot{\mathrm{A}}^{3}, \quad l_{c}=\left[1.54+1.26\left(n_{c}-\right.\right.
$$

1)] $\dot{\mathrm{A}}$ is the maximum chain length, and $n_{c}$ is the number of carbon atoms in the hydrocarbon chain. $A_{\min }$ refers to the property associated with the surfactant monolayer at the air/ water interface. $P$ determines the geometry of micelles and denotes the minimum sized aggregates in solution, which reduces the Gibbs free energy of micellization.

The maximum surface excess concentration at the air/ methanol-water interface $\Gamma_{\max }$, area occupied per surfactant molecule $\left(A_{\min }\right)$ at the air/methanol-water interface, surface pressure at the $\mathrm{cmc}\left(\pi_{\mathrm{cmc}}\right)$, standard free energy interfacial adsorption $\left(\Delta G_{\text {ads }}^{\mathrm{o}}\right)$, and packing parameters $(P)$ of CTAB solutions in water, $0.10,0.20,0.30$, and 0.40 volume fractions of methanol-water at 298.15, 308.15, 318.15, and $323.15 \mathrm{~K}$, were calculated, and the values are displayed in Table 1.

Chakraborty et al. [36] calculated the $\mathrm{cmc}(\mathrm{mM})$ of $\mathrm{CTAB}$ at $25^{\circ} \mathrm{C}$ by $(0.959)$ conductivity and by $(0.883)$ tensiometry with $\gamma_{\mathrm{cmc}}=33.1$. Our data of $\mathrm{CTAB}$ at $25^{\circ} \mathrm{C}$ in water regarding $\mathrm{cmc}$ is $0.97 \mathrm{mM}$ from tensiometry with $\gamma_{\mathrm{cmc}}=33.4$, which almost matched with literature [36].

Similarly, Shah et al. [21, 37] calculated the cmc of CTAB at $298.15 \mathrm{~K}$ in water $(0.97 \mathrm{mM})$ by tensiometry, $\Delta G_{\mathrm{m}}^{\mathrm{o}}=-49.90 \mathrm{~kJ} \cdot \mathrm{mol}^{-1}, \quad \Gamma_{\max } \times 10^{6}=3.01\left(\mathrm{~mol} \cdot \mathrm{m}^{-2}\right)$, $A_{\text {min }}=-54.98 \dot{\mathrm{A}}^{2}$ molecule ${ }^{-1}, \pi_{\mathrm{cmc}}=33.59$, packing parameter $(P)=0.38$, and $\left(\Delta G_{\text {ads }}^{\mathrm{o}}\right)=-61.02 \mathrm{~kJ} \cdot \mathrm{mol}^{-1}$. These data almost matched with our system (Table 1 ). When the temperature was increased, the $\mathrm{cmc}$ increased from $0.97 \mathrm{mM}$ at $298.15 \mathrm{~K}$ to $1.14 \mathrm{mM}$ at $308.15 \mathrm{~K}$ (Table 1 ). The increase in $\mathrm{cmc}$ with temperature is due to the smaller probability of hydrogen bond formation [38].

The relative permittivity of the medium decreases when methanol is added to water because methanol has a lower relative permittivity than pure water and a lower relative 
TABle 1: Critical micelle concentration $(\mathrm{cmc})$, maximum surface excess concentration $\left(\Gamma_{\max }\right)$, area occupied by surfactant molecule $\left(A_{\text {min }}\right)$, surface pressure at the $\mathrm{cmc}\left(\pi_{\mathrm{cmc}}\right)$, packing parameters $(P)$, and free energy of adsorption $\left(\Delta \mathrm{G}_{\mathrm{ads}}^{\mathrm{o}}\right)$.

\begin{tabular}{|c|c|c|c|c|c|c|c|}
\hline$T(\mathrm{~K})$ & Volume fractions of methanol & $\mathrm{cmc}(\mathrm{mM})$ & $\Gamma_{\max } 10^{6}\left(\mathrm{~mol} \cdot \mathrm{m}^{-2}\right)$ & $A_{\min }\left(\AA^{2}\right.$ molecule $\left.^{-1}\right)$ & $\pi_{\mathrm{cmc}}\left(\mathrm{m} \cdot \mathrm{Nm}^{-1}\right)$ & $P$ & $\Delta G_{\mathrm{ads}}^{\mathrm{o}}\left(\mathrm{kJ} \cdot \mathrm{mol}^{-1}\right)$ \\
\hline \multirow{5}{*}{298.15} & 0.0 & 0.97 & 3.01 & 54.98 & 33.59 & 0.38 & -63.40 \\
\hline & 0.1 & 1.22 & 2.10 & 78.73 & 18.09 & 0.26 & -57.01 \\
\hline & 0.2 & 1.78 & 1.72 & 96.33 & 10.23 & 0.21 & -51.46 \\
\hline & 0.3 & 3.55 & 0.94 & 175.71 & 8.07 & 0.11 & -47.32 \\
\hline & 0.4 & 6.43 & 0.77 & 213.26 & 4.32 & 0.09 & -39.02 \\
\hline \multirow{5}{*}{308.15} & 0.0 & 1.14 & 2.83 & 58.49 & 32.91 & 0.36 & -63.9 \\
\hline & 0.1 & 1.42 & 1.82 & 91.14 & 18.07 & 0.23 & -57.55 \\
\hline & 0.2 & 1.88 & 1.55 & 107.07 & 11.50 & 0.19 & -52.16 \\
\hline & 0.3 & 4.45 & 0.76 & 217.96 & 8.75 & 0.09 & -48.63 \\
\hline & 0.4 & 7.34 & 0.62 & 267.33 & 5.06 & 0.07 & -40.42 \\
\hline \multirow{5}{*}{318.15} & 0.0 & 1.22 & 2.22 & 74.65 & 33.52 & 0.28 & -64.22 \\
\hline & 0.1 & 1.71 & 1.72 & 96.33 & 20.04 & 0.21 & -57.93 \\
\hline & 0.2 & 2.56 & 1.41 & 117.62 & 12.82 & 0.17 & -52.54 \\
\hline & 0.3 & 5.66 & 0.68 & 243.45 & 8.45 & 0.08 & -47.07 \\
\hline & 0.4 & 7.81 & 0.52 & 315.62 & 6.03 & 0.06 & -41.60 \\
\hline \multirow{5}{*}{323.15} & 0.0 & 1.32 & 2.17 & 76.39 & 33.79 & 0.27 & -64.37 \\
\hline & 0.1 & 1.91 & 1.60 & 103.78 & 21.87 & 0.20 & -58.66 \\
\hline & 0.2 & 2.73 & 1.22 & 136.08 & 14.05 & 0.15 & -53.02 \\
\hline & 0.3 & 5.89 & 0.57 & 288.61 & 9.29 & 0.07 & -49.31 \\
\hline & 0.4 & 7.32 & 0.43 & 382.66 & 6.89 & 0.05 & -42.30 \\
\hline
\end{tabular}

permittivity decreases the hydrophobic interaction and increases the $\mathrm{cmc}[20]$.

The data show that $\Gamma_{\max }$, as well as $\pi_{\mathrm{cmc}}$ values, decreases with an increase in the volume fraction of methanol at a particular temperature indicating less population of surfactant molecules at the interface with the addition of methanol. Our decrease in data of $\Gamma_{\max }$ with the addition of methanol was matched with the literature [25]. With the increase in temperature, $\Gamma_{\max }$ values decrease (Table 1). The reason may be due to the enhanced molecular thermal agitation at higher temperatures [39]. Such trends were also observed in the previous study [20].

With the increasing temperature, $\pi_{\mathrm{cmc}}$ is increased. The reason may be that $\pi_{\mathrm{cmc}}$ is a measure of the efficiency of the surfactant to lower $\gamma$ of water [40].

However, $A_{\min }$ values increase with the addition of methanol that denotes the surfactant molecules occupy more surface areas at the air/methanol-water interface. Such a trend was also observed by Mandal et al. in 2019 [25].

With the increase in temperature, $A_{\min }$ increases (Table 1). The reason may be due to the thermal molecular motion at high temperatures [41].

Negative values of $\Delta G_{\mathrm{ads}}^{\mathrm{o}}$ indicate that the adsorption of surfactant molecules on the surface is spontaneous, and this phenomenon is more spontaneous than micellization due to a larger negative value than $\Delta G_{\mathrm{m}}^{o}$. The values of $\Delta G_{\mathrm{ads}}^{\mathrm{o}}$ became less negative with the increase in the volume fraction of methanol at a particular temperature that shows less spontaneity of adsorption of surfactant molecules on the surface.

On increasing the temperature, $\Delta G_{\text {ads }}^{o}$ values become more negative, which indicates the higher spontaneity of adsorption of $\mathrm{CTAB}$ molecules on the surface. Such trends were also observed in the literature [20].
Equation (5) is used to describe the temperature dependence of $\Delta G_{\mathrm{m}}^{o}$. The plot of $\Delta G_{\mathrm{m}}^{o} / T$ with $1 / T$ shows the linear variation in water, $0.10,0.20,0.30$, and 0.40 volume fractions of methanol-water (Figure 2). Here, $\Delta G_{\mathrm{m}}^{o}$ has been taken from the earlier calculations [37]. Negative value $\Delta G_{\mathrm{m}}^{o}$ indicates that micellization is spontaneous. This spontaneity decreases with an increase in the volume fraction of methanol; subsequently, micellization is less favorable. The molecular interactions between the amphiphiles needed to micelle are agitated by the presence of methanol, which becomes more pronounced with the addition of the volume fraction of methanol.

3.2. Variation in the Slope $(d \gamma / d \log C)$ of Surface Tension Curve with the Volume Fraction of Methanol. The slope $(\mathrm{d} \gamma / \operatorname{d} \log C)$ of the surface tension curve (Figure 3$)$, where $C$ is the concentration of the CTAB solution in water and the methanol-water mixture at 298.15 to $323.15 \mathrm{~K}$, gives various information about the surface properties of the surfactant solution [23]. It is seen that the slopes increase with the increase in methanol and also increase with temperature. As it is mentioned in equation (1) that $\Gamma_{\max }$ is calculated from the slope $\mathrm{d} \gamma / \mathrm{d} \log C$, the more negative the value of the slope, the larger the values of surface properties. Figure 3 shows that, with the increase in methanol content, values of slope are becoming less negative. Additionally, this slope indicates how pronounced the $\mathrm{cmc}$ can be located in the plot of $\log C$ vs $\gamma$. In the case of the aqueous solution, the value of the slope is maximum, indicating that it is very easy to locate the $\mathrm{cmc}$, whereas in the case of 0.4 volume fraction of methanolwater, the slope is minimum, indicating that it is difficult to locate the $\mathrm{cmc}$. This makes the limitation of our work up to 0.4 volume fraction of methanol only [37]. As methanol 


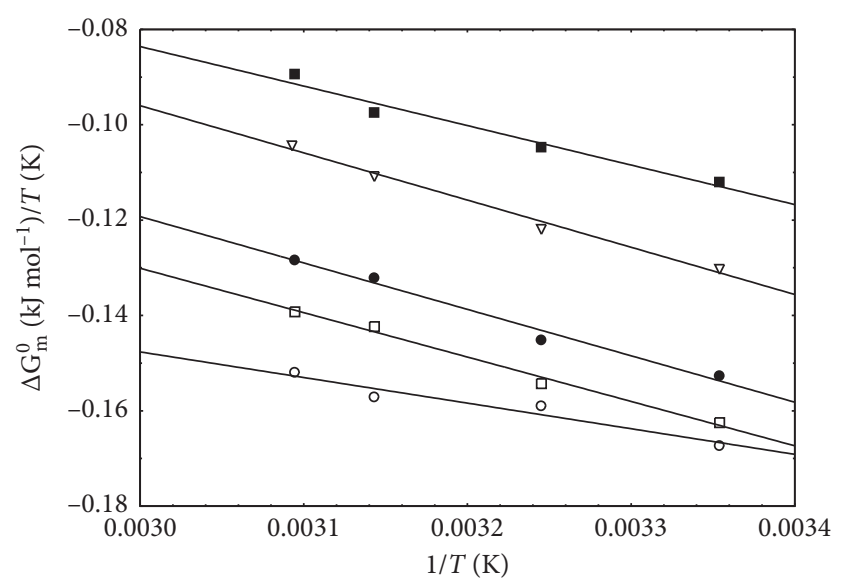

- 0.40 methanol

$\nabla 0.30$ methanol

ㅁ 0.10 methanol

Figure 2: Plot of $\Delta G_{\mathrm{m}}^{o} / T$ with $1 / T$ of CTAB solution in pure water and different volume fractions of methanol-water.

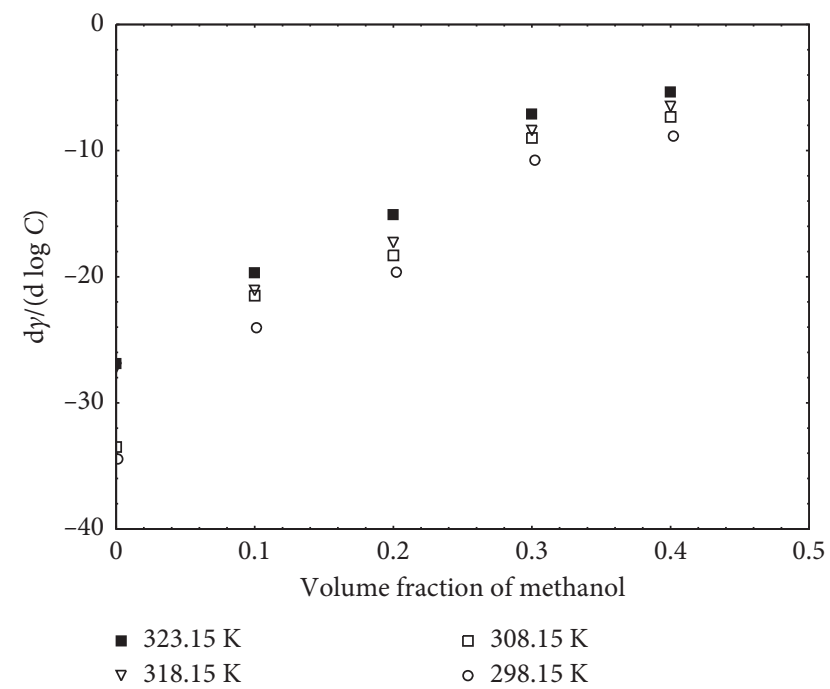

Figure 3: Variation in slope $\mathrm{d} \gamma / \mathrm{d} \log C$ with volume fractions of methanol for CTAB solutions.

increases, the formation of the micelle is very difficult to predict due to the solubilization of micelles [34, 42]. However, in a mixed surfactant system of cationic rich and anionic rich, 0.3 volume fraction of methanol was limited due to solubilization of micelles [43].

\subsection{Correlation of $\gamma_{o} / \gamma_{c m c}$ with the Volume Fraction of} Methanol. In 2012, Mukhim and Ismail [44] assumed that the ratio $\gamma_{o} / \gamma_{\mathrm{cmc}}$ can be utilized to discuss the solvophobic effect [45]. Figure 4 shows variation in $\gamma_{o} / \gamma_{\mathrm{cmc}}$ with the volume fraction of methanol for CTAB at 298.15 to $323.15 \mathrm{~K}$. The values of $\gamma_{o} / \gamma_{\mathrm{cmc}}$ decrease with the increase in methanol and but increase with temperature. The curves indicate that the solvophobic effect decreases with increasing in methanol content.

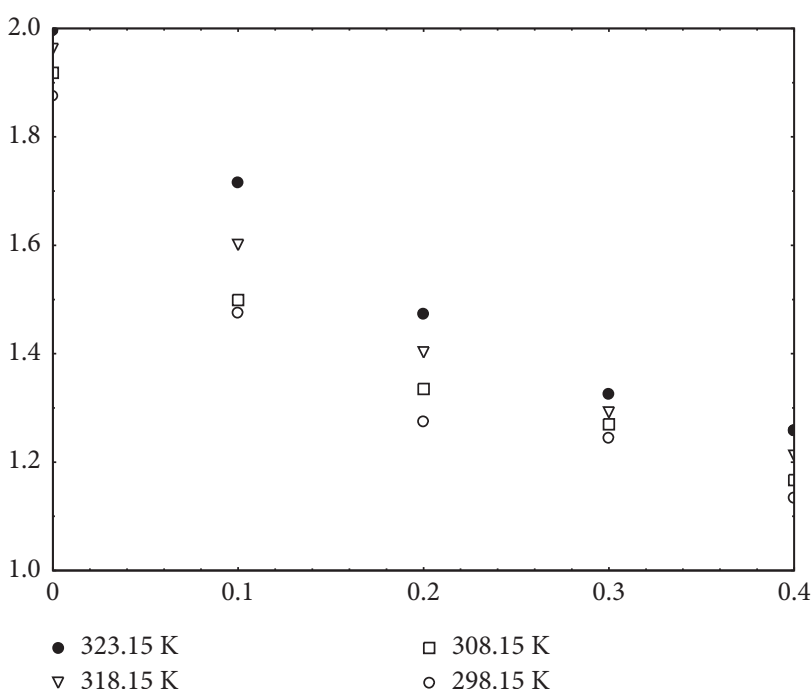

FIgURE 4: Variation in $\gamma_{o} / \gamma_{\mathrm{cmc}}$ CTAB solution with the volume fraction of methanol: square $(298.15 \mathrm{~K})$, triangles $(308.15 \mathrm{~K})$, closed circles $(318.15 \mathrm{~K})$, and closed squares $(323.15 \mathrm{~K})$.

3.4. Correlation of $\Delta G_{a d s}^{o}$ with Temperature. Gibbs energies of adsorption of CTAB in water and in a mixture of methanol-water show unique variation at different temperatures (Figure 5). It can be seen from Table 1 that the values of $\Delta G_{\text {ads }}^{o}$ are all negative, indicating adsorption of amphiphiles is spontaneous at all investigated temperatures and volume fractions of methanol. However, the presence of methanol makes the adsorption less easy because methanol acts as a cosolvent and reduces the surface tension by adsorbing on the air/water interface [20]. $\Delta G_{\text {ads }}^{o}$ linearly decreases with the increase in temperature whereas it increases with the increase in methanol. It was found that there is a systematic variation in the slopes with temperatures. Therefore, it will be easier for us to calculate the standard Gibbs energies of adsorption. Such variation in $\Delta G_{\text {ads }}^{\mathrm{o}}$ with solvent composition was also seen in the literature [38].

3.5. Correlation of $p C_{20}$ with the Volume Fraction of Methanol. When we plot $\gamma$ versus $\log C$, the minimum concentration of the surfactant can be obtained. The bulk liquid phase concentration of surfactant required to lower the surface tension of the solvent by $20 \mathrm{mN} / \mathrm{M}$ is a good measure of the efficiency of the adsorption of the surfactant (or surfactant efficiency). Hence, the negative logarithm of the concentration of surfactant in the bulk phase necessary to make a $20 \mathrm{mN} / \mathrm{M}$ diminution in $\gamma$ of the solvent is the efficiency of adsorption $\left(p C_{20}\right)$.

The standard adsorption Gibbs free energy change, $\Delta G_{\text {ads }}^{\mathrm{o}}$, involved in the transfer of the surfactant molecule from the interior of the bulk liquid phase to the interface. We have the relation of $p C_{20}$ by use of the Langmuir and Szyszkowski equations [46] as

$$
p C_{20}=-\left(\frac{\Delta G_{\mathrm{ads}}^{\mathrm{o}}}{2.303 R T}+1.74+\frac{20}{2.303 R T \Gamma_{\max }}\right) .
$$




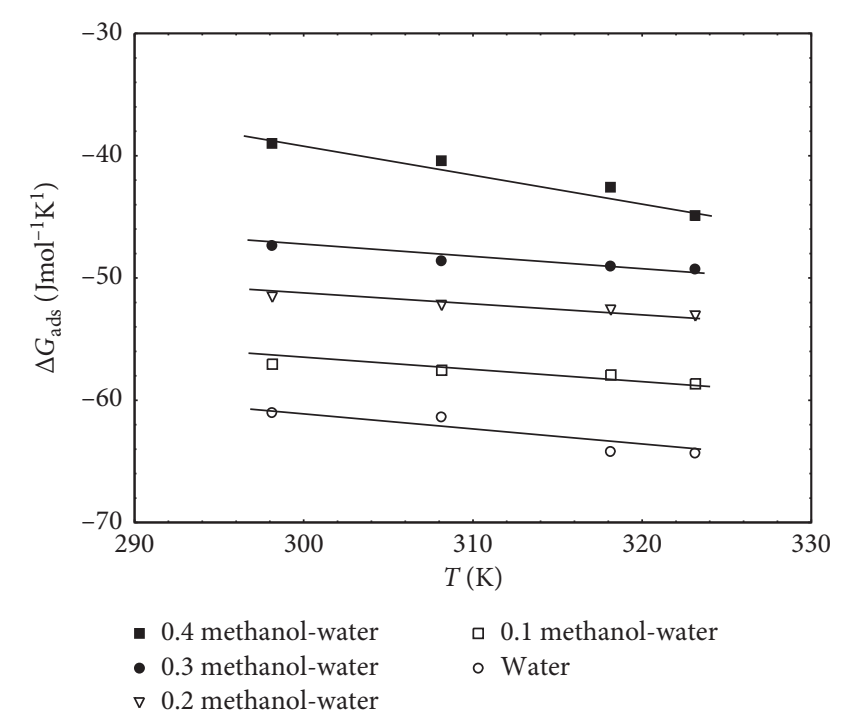

FIgURE 5: Variation in $\Delta G_{\text {ads }}^{\mathrm{o}}$ of CTAB solution with temperature.

The value of $p C_{20}$ at $25^{\circ} \mathrm{C}$ calculated using equation (8) is 7.43 , which does not match with the literature values of 3.67 [47] and 3.15 [46]. According to equation (8), $p C_{20}$ is calculated using the values of $\Delta G_{\text {ads }}^{o}$ and $\Gamma_{\max }$ at a constant temperature. Likewise, $\Delta G_{\text {ads }}^{\mathrm{o}}$ is calculated using the value of $\Delta G_{\mathrm{m}}^{o}$, and the latter quantity depends on the degree of ionization $(\alpha)$ and $\mathrm{cmc}$. For example, $\alpha=0.56$ and $\mathrm{cmc}=0.00095 \mathrm{M}$; then, $\Delta G_{\mathrm{m}}^{o}$ turns out to be $-27.42 \mathrm{~kJ} \cdot \mathrm{mol}^{-1}$, and obviously, we can determine $\Delta G_{\text {ads }}^{o}$. However, Niranjan et al. in 2011 [48] calculated $\Delta G_{\text {ads }}^{o}$ without using $\pi_{c \mathrm{~cm}}$ [46] obtained as $-37.05 \mathrm{~kJ} \cdot \mathrm{mol}^{-1}$ which is on the right trends in comparison to their $\Delta G_{\mathrm{m}}^{o}$. By using $\Delta G_{\mathrm{ads}}^{\mathrm{o}}$ and $\Gamma_{\text {max }}$, we obtained the value of $p C_{20}$ from equation (9) equal to 3.11 , which is almost matched with the literature value of 3.15 for CTAB at $25^{\circ} \mathrm{C}$ [48].

It was surprising for us, so the data of $\Delta G_{\text {ads }}^{o}$ and $\Gamma_{\max }$ were taken randomly from the recent work of Zhang et al. [47] and used in equation (8). The value of $p C_{20}$ was calculated to be 7.44 which was high in comparison to other literatures and searched more details on the work of Zhang et al. [47] and found that $\alpha=0.38$ and $\mathrm{cmc}=0.00084 \mathrm{M}$ and then $\Delta G_{\mathrm{m}}^{o}=-47.04 \mathrm{~kJ} \cdot \mathrm{mol}^{-1}$ and $\Delta G_{\mathrm{ads}}^{\mathrm{o}}=-59.6 \mathrm{~kJ} \cdot \mathrm{mol}^{-1}$ which matched with our data. Therefore, we used the equation from the same literature [47] as

$$
p C_{20}=\frac{\pi_{\mathrm{cmc}}-20}{2.303 n R T \Gamma_{\max }}-\log \mathrm{cmc} \text {, }
$$

and found the value 3.43 of $p C_{20}$ for $\mathrm{CTAB}$ at $25^{\circ} \mathrm{C}$ which matched almost with the literature. For $\mathrm{CTAB}$ at $25^{\circ} \mathrm{C}$, Javadian et al. [49] calculated the value of $p C_{20}=0.8$, using $\alpha=0.30$ without using $\Delta G_{\mathrm{ads}}^{\mathrm{o}}$. This invigorated us to use equation (9) for the calculation of values of $p C_{20}$.

Thus, we can conclude that equation (8) is valid only if $\alpha$ is high and $\Delta G_{\text {ads }}^{o}$ is less negative, whereas equation (9) is applicable if $\alpha$ is low and $\Delta G_{\text {ads }}^{\mathrm{o}}$ is more negative. Moreover, equation (9) is even useful if we do not have $\Delta G_{\text {ads }}^{o}$, but there is the necessity of $\pi_{\mathrm{cmc}}$ and cmc.

The plot $p C_{20}$ of CTAB in water and methanol-water mixed solvent media (Figure 6) gives very interesting

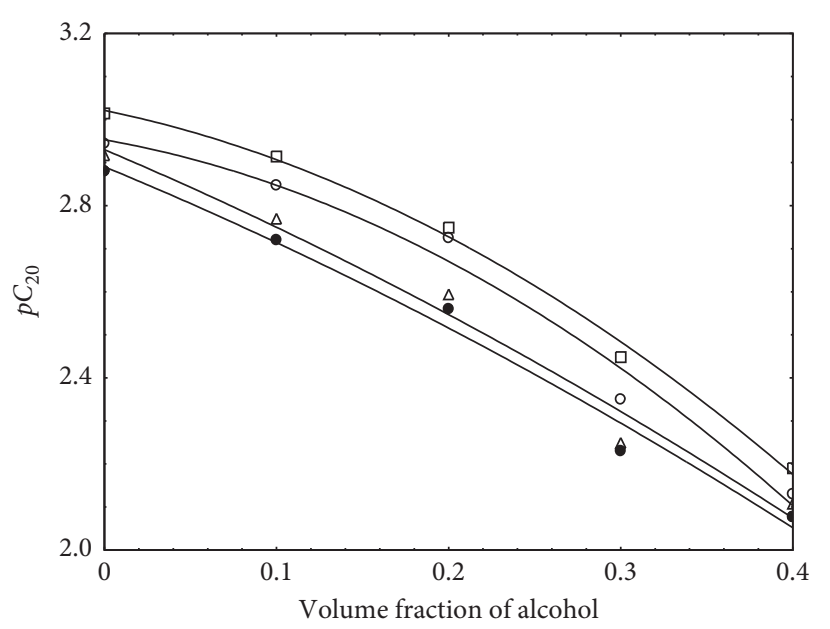

FIgURE 6: Variation in $p C_{20}$ CTAB with the volume fraction of methanol: squares $(298.15 \mathrm{~K})$, circles $(308.15 \mathrm{~K})$, triangles $(318.15 \mathrm{~K})$, and closed circles $(323.15 \mathrm{~K})$.

information. Data show that $p C_{20}$ has a maximum value in the water at $298.15 \mathrm{~K}$ which decreases with increasing volume fractions of methanol at the same temperature. This signifies that the adsorption efficiency decreases in the presence of methanol, which is attributed due to a decrease in the solvophobicity of the medium.

The values of $p C_{20}$ are least for 0.4 volume fraction of methanol-water at the respective temperatures. It is interesting to note that, in the case of 0.4 volume fraction of methanol, change in surface tension is in a narrow range (as shown in Figure 1). In such case, adsorption efficiency is least significant. This is further justified by the fact that the formation of micelles at 0.4 and beyond the volume fraction of methanol is not significant enough to discuss surface properties [34].

$p C_{20}$ of CTAB in the water at $298.15 \mathrm{~K}$ shows higher values in comparison with higher temperatures and methanol-water mixed solvent media. This suggests that elevated temperature is not favorable for micellization, corresponding to the $\mathrm{cmc}$ trends [50]. The convex nature of the curves decreases for $298.15 \mathrm{~K}$ and $308.15 \mathrm{~K}$ with the addition of methanol, whereas the convex nature of the curves decreases and looks almost linear for $318.15 \mathrm{~K}$ and $323.15 \mathrm{~K}$ with the addition of methanol (Figure 6).

3.6. Correlation of $\Delta S_{a d s}^{o}$ with the Volume Fraction of Methanol. As we know from the literature [51], the standard entropy of adsorption $\left(\Delta S_{\text {ads }}^{\mathrm{o}}\right)$ can be calculated if the standard Gibbs free energy of adsorption is known:

$$
\begin{gathered}
\Delta G_{\mathrm{ads}}^{\mathrm{o}}=\Delta H_{\mathrm{ads}}^{\mathrm{o}}-T \Delta S_{\mathrm{ads}}^{\mathrm{o}}, \\
\Delta S_{\mathrm{ads}}^{\mathrm{o}}=-\left(\frac{\partial \Delta G_{\mathrm{ads}}^{\mathrm{o}}}{\partial T}\right) .
\end{gathered}
$$

If standard enthalpy of adsorption $\left(\Delta H_{\mathrm{ads}}^{\mathrm{o}}\right)$ is constant over the temperature range studied, $\Delta G_{\text {ads }}^{o}$ is calculated using equation (6) and the values are presented in Table 1 . It is 
reported that, for ionic surfactants, the $\Delta H_{\text {ads }}^{\mathrm{o}}$ values determined based on $\Delta G_{\text {ads }}^{o}$ values by using $\Delta S_{\text {ads }}^{o}$ calculated from equation (11) are almost less possible. Therefore, $\Delta H_{\text {ads }}^{\mathrm{o}}$ values are determined using $\Delta G_{\mathrm{ads}}^{\mathrm{o}} / 2[52,53]$, and $\Delta S_{\text {ads }}^{\mathrm{o}}$ values are calculated using equation (10). Figure 7 shows that the values of $\Delta S_{\text {ads }}^{o}$ are all positive under all conditions. $\Delta S_{\text {ads }}^{o}$ increases with the increase in methanol, and the concave variation in $\Delta S_{\text {ads }}^{\circ}$ with the volume fraction of methanol was observed (Figure 7). The increase in the positive values of $\Delta S_{\text {ads }}^{o}$ with the increase in the volume fraction of methanol indicates that the surfactant molecules prefer more scattered forms.

3.7. Correlation of $\Delta H_{\text {ads }}^{o}$ with the Volume Fraction of Methanol. Figure 8 shows that the values $\Delta H_{\text {ads }}^{\circ}$ are all negative except 0.4 volume fractions of methanol. This shows that adsorption is exothermic. $\Delta H_{\text {ads }}^{\circ}$ increases with the increase in methanol, and the concave variation of $\Delta H_{\mathrm{ads}}^{\mathrm{o}}$ with the volume fraction of methanol was observed. The addition of methanol decreases the cohesive energy of the solution. The decrease in the cohesive energy is maximum in the case of 0.4 volume fraction of methanol. Therefore, the dispersion of surfactant molecules takes place. The variation in the curve is similar to the variation in the curve of $\Delta S_{\mathrm{ads}}^{\mathrm{o}}$ with the volume fraction of methanol (Figure 7).

3.8. Correlation of Packing Parameter with the Volume Fraction of Methanol. The value of the packing parameter $(P)$ gives the shape of the micelle. When $P$ values are within $0-0.33$, then the shape of the micelle is spherical, whereas for the cylindrical shape of the micelle, the values of $P$ should be between 0.33 and 0.5 . In some cases, we can find the lamellar shape of the micelle; under such conditions, the values of $P$ will be between 0.5 and 1 .

In aqueous solution, $\mathrm{CTAB}$ forms cylindrical micelles. The variation in the packing parameter of $\mathrm{CTAB}$ with the volume fraction of methanol shows a unique variation. The $P$ values of CTAB monotonously decrease like a concave curve with the addition of methanol, whereas for the temperatures $(308.15 \mathrm{~K}$ and $323.15 \mathrm{~K}$ ), the $P$ values of CTAB decrease sharply in linear fashion, as shown in Figure 9. In all systems, the $P$ values of $\mathrm{CTAB}$ decrease with temperature. This may be because of inducing vibrational, rotational, and translational oscillations with wreaking of the binding forces of the CTAB system [39]. Such behavior was also noticed in a previous study [20]. The $P$ values of CTAB decrease with the addition of methanol because of leading to a decrease in the solubilization capacity [54]. Such behavior was also seen in the literature [55].

3.9. Correlation of $\Delta G_{\text {eff }}^{o}$ with the Volume Fraction of Methanol. The difference between $\Delta G_{\mathrm{ads}}^{\mathrm{o}}$ and $\Delta G_{m}^{o}$ is called the effective Gibbs free energy $\left(\Delta G_{\text {eff }}^{o}\right)$. The curve of $\Delta G_{\text {eff }}^{o}$ sharply increases with convex variation with the addition of methanol and becomes a linear curve for CTAB at $323.15 \mathrm{~K}$. With the increase in temperature, the curve of $\Delta G_{\text {eff }}^{o}$ decreases, as shown in Figure 10. It is also observed that the values of $\Delta G_{\text {eff }}^{o}$ decrease with an increase in the head group

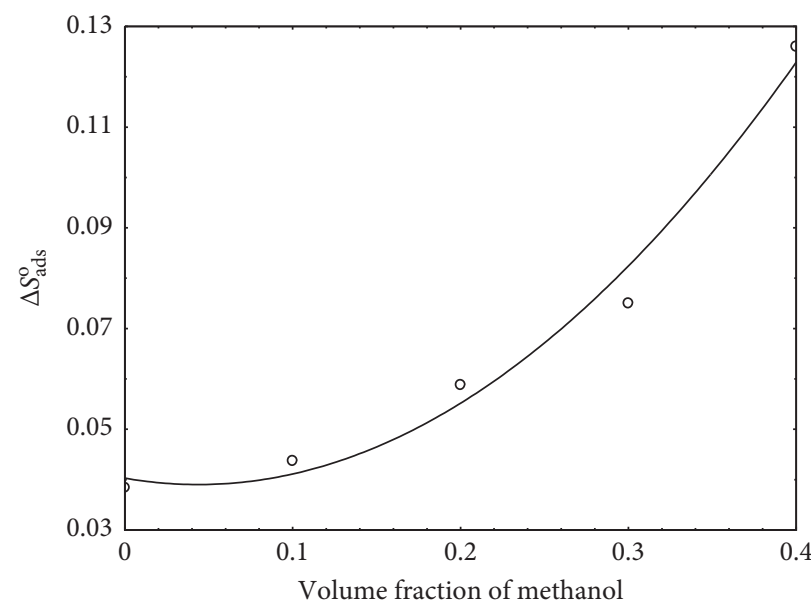

Figure 7: Variation in $\Delta S_{\text {ads }}^{o}$ with volume fraction of methanol.

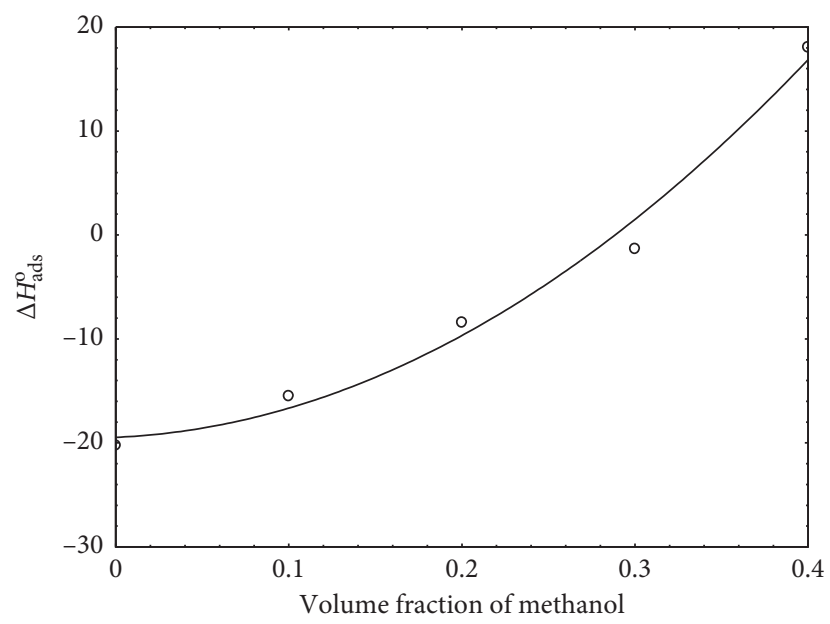

FIgURE 8: Variation in $\Delta H_{\text {ads }}^{\mathrm{o}}$ with volume fraction of methanol.

polarity of $\mathrm{CTAB}$, indicating that aggregation is more favored than the adsorption process and also less energy is required for the aggregation process [56].

3.10. Correlation of $N$ with the Volume Fraction of Methanol. The number of molecules present in a micelle once the $\mathrm{cmc}$ is reached is called an aggregation number $(N)$ [57].

146 was the aggregation number for CTAB in water at $25^{\circ} \mathrm{C}$ in the literature [58]. It was also noticed that the reported value of $N$ was higher because Goddard found $N=89$ at $20^{\circ} \mathrm{C}$ [59].

Similarly, Hartley [60] in his classical monograph mentioned spherical-shaped micelles with aggregation numbers in the range of 50 to 100 for CTAB solutions.

We know that the shape of a micelle is related to its size. The size is usually expressed in terms of an aggregation number that can be determined in different ways like light scattering [61, 62] and diffusion [63] and sedimentation rates in ultracentrifugation $[64,65]$. Other scattering techniques $[66,67]$ such as X-ray scattering, quasielastic light scattering, 


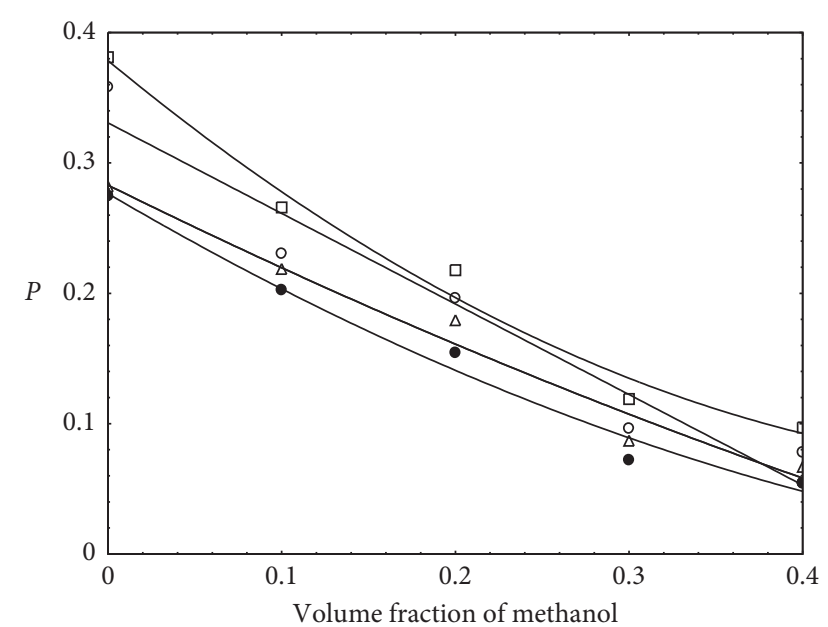

Figure 9: Variation in $P$ with the volume fraction of methanol: squares $(298.15 \mathrm{~K})$, circles $(308.15 \mathrm{~K})$, triangles $(318.15 \mathrm{~K})$, and closed circles $(323.15 \mathrm{~K})$.

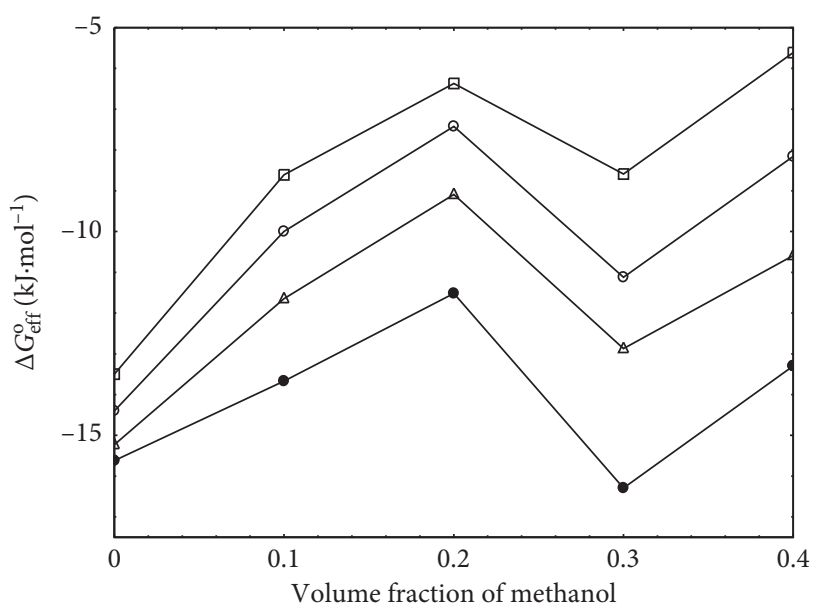

FIGURE 10: Variation in $\Delta G_{\text {eff }}^{o}$ with the volume fraction of methanol: squares $(298.15 \mathrm{~K})$, circles $(308.15 \mathrm{~K})$, triangles $(318.15 \mathrm{~K})$, and closed circles $(323.15 \mathrm{~K})$.

and neutron scattering have also been used for the determination of aggregation numbers. Lindman used NMR techniques $[68,69]$ to determine aggregation numbers of ionic and nonionic surfactants. The use of fluorescence probes which was a relatively convenient technique became popular [61]. Till now, both static and dynamic fluorescence techniques can be used to obtain aggregation numbers, and the latter is considered to be superior to the former [61].

The aggregation number is generally estimated experimentally by NMR and fluorescence measurements, and they are involved in concentration determination or the number of micelles together with the total amount of surfactants in the micellar form which is used to estimate the aggregation number [70]. However, we have determined here the aggregation number by using the simple equation with varying $A_{\min }$ described in the literature [71] as

$$
N=\frac{4 \pi l_{c}^{2}}{A_{\min }},
$$

where $A_{\min }$ is the area occupied by per surfactant molecule and $l_{\mathrm{c}}=20.44 \AA$ (hydrophobic tail length).

Our aggregation number almost matched with the literature [72] for $\mathrm{CTAB}$ in water at $25^{\circ} \mathrm{C}$. It is interesting to see the variation in $N$ with the volume fraction of methanol (Figure 11). $N$ of CTAB varies concave curve on decreasing monotonously with the addition of methanol. On increasing temperature from $298.15 \mathrm{~K}$ to $308.15 \mathrm{~K}$, the concave curves are observed, and further increasing temperature, the curve tends to change into a lower value somehow linearly by decreasing sharply with the volume fraction of methanol $(318.15 \mathrm{~K}$ and $323.15 \mathrm{~K})$. The reason for the decrease in $N$ with increase in temperature may be due to the dissociation of the intra-micellar excimers play the role for the complete interpretation of the results at high temperature [73].

3.11. The Relation between $A_{\min }$ and $\pi_{c m c} . \pi_{\mathrm{cmc}}$ is a measure of the cohesive force in the surfactant film, whereas $A_{\text {min }}$ describes the "orientation" of the surfactant's molecule in an aqueous solution. $\pi_{\mathrm{cmc}}$ decreases with increasing temperature and methanol (Table 1), whereas $A_{\min }$ increases with increasing temperature and methanol (Table 1). With the increase in $A_{\mathrm{min}}$, the $\pi_{\mathrm{cmc}}$ value of CTAB decreases sharply in linear fashion with the addition of methanol up to 0.2 volume fraction and then decreases monotonously until 0.4 volume fraction of methanol for $318.15 \mathrm{~K}$ and $323.15 \mathrm{~K}$. With the temperatures $298.15 \mathrm{~K}$ and $308.15 \mathrm{~K}$ increase in $A_{\min }$, the $\pi_{\mathrm{cmc}}$ value of CTAB decreases sharply in linear fashion with the addition of methanol up to 0.2 volume fraction and then decreases monotonously until 0.3 volume fraction of methanol and then decreases sharply up to 0.4 volume fraction of methanol (Table 1). The pattern of our variation in $\pi_{\mathrm{cmc}}$ versus $A_{\mathrm{min}}$ looks like the curve of $\pi_{\mathrm{cmc}}$ versus $A_{\mathrm{min}}$ of SDCS in the absence and presence of polymer [74]. The same pattern of the curve of CTAB was observed in the literature [75].

We also plot the curve of $\pi_{\mathrm{cmc}} A_{\min }$ with $\pi_{\mathrm{cmc}}$ : the highest curve is at temperature $323.15 \mathrm{~K}$ and then decreases for $318.15 \mathrm{~K}, 308.15 \mathrm{~K}$, and $298.15 \mathrm{~K}$ simultaneously (Figure S1). With the increase in methanol, at first, the curve increased to 0.1 volume fraction of methanol and then decreased until 0.2 volume fraction of methanol and again increases continuously (Figure S1).

The plot of area $\left(A_{\mathrm{min}}\right)$ against $1 / \pi_{\mathrm{cmc}}$ gives interesting results (Figure S2). At $298.15 \mathrm{~K}$ and $308.15 \mathrm{~K}$, the curves increase with convex nature with the addition of methanol, whereas for $318.15 \mathrm{~K}$ and $323.15 \mathrm{~K}$, the curves increase with concave nature. The highest curve is at temperature $323.15 \mathrm{~K}$ and then decreases for $318.15 \mathrm{~K}, 308.15 \mathrm{~K}$, and $298.15 \mathrm{~K}$ simultaneously (Figure S2).

3.12. Correlation of $\Gamma / \Gamma_{\max }$ with Volume Fraction of Methanol. Applying the Frumkin isotherm equation provided in the literature [46], it confirmed the concentration value at which $\gamma$ of water is minimized by $20 \mathrm{mN} / \mathrm{m}$, and the 


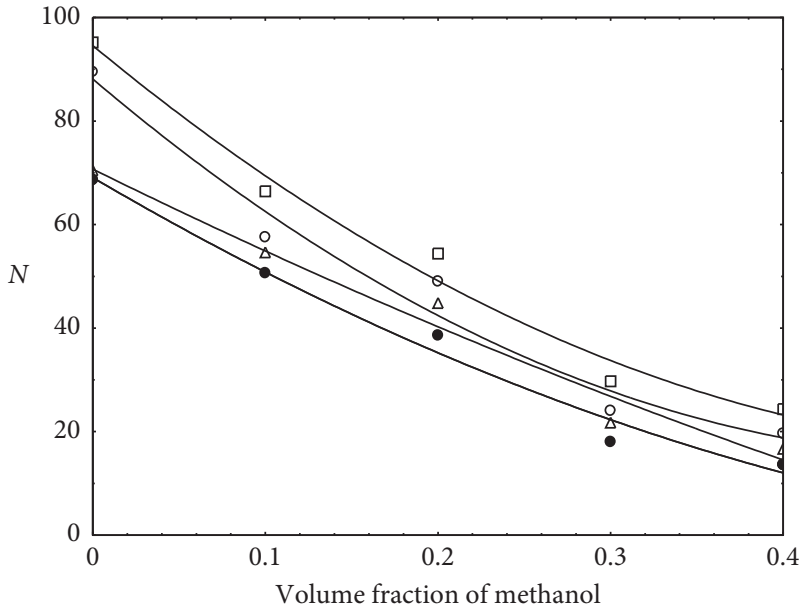

Figure 11: Variation in $N$ CTAB with the volume fraction of methanol: triangles $(298.15 \mathrm{~K})$, circles $(308.15 \mathrm{~K})$, squares $(318.15 \mathrm{~K})$, and circles $(323.15 \mathrm{~K})$.

ratio of the Gibbs surface excess concentration $(\Gamma)$ to the maximal $\left(\Gamma_{\max }\right)$ is within 0.84 to 0.999 .

We have calculated the $\Gamma / \Gamma_{\max }$ values for CTAB systems from the equation used in the literature [46]:

$$
\pi_{\mathrm{cmc}}=-R T \Gamma_{\max } \ln \left(1-\frac{\Gamma}{\Gamma_{\max }}\right) .
$$

Our values of $\Gamma / \Gamma_{\max }$ for the CTAB system perfectly matched within the range of the literature [46].

On adding methanol, the values of $\Gamma / \Gamma_{\max }$ decrease sharply for the CTAB system at $298.15 \mathrm{~K}$ and $308.15 \mathrm{~K}$ and monotonously decrease at $318.15 \mathrm{~K}$ and $323.15 \mathrm{~K}$ up to 0.2 volume fraction of methanol (Figure S3).

The values of $\Gamma / \Gamma_{\max }$ increase sharply for the $\mathrm{CTAB}$ system at $298.15 \mathrm{~K}$ and $308.15 \mathrm{~K}$ and monotonously increase at $318.15 \mathrm{~K}$ and $323.15 \mathrm{~K}$ up to 0.3 volume fraction of methanol (Figure S3), and finally, the curves decrease sharply for the CTAB system at $298.15 \mathrm{~K}$ and $308.15 \mathrm{~K}$ and monotonously decrease at $318.15 \mathrm{~K}$ and $323.15 \mathrm{~K}$ (Figure S3). The highest curve is for $323.15 \mathrm{~K}$, and the lowest is for $298.15 \mathrm{~K}$ (Figure S3). But the values of $\Gamma / \Gamma_{\max }$ for water, 0.1, $0.2,0.3$, and 0.4 volume fraction of methanol are more than 0.9 for all investigated temperatures. The exceptional case is for $298.15 \mathrm{~K}$ of 0.4 volume fraction of methanol.

3.13. Correlation of $\mathrm{cmc} / C_{20}$ with the Volume Fraction of Methanol. When the surface is saturated and $\gamma$ of the solvent is minimized by $20 \mathrm{mN} / \mathrm{M}$, then for the linear portion of the plot, the Gibbs adsorption equation becomes [46] as

$$
\pi_{\mathrm{cmc}} \approx 20+2.3 n R T \Gamma_{\max } \log \left(\frac{\mathrm{cmc}}{C_{20}}\right) \text {. }
$$

This equation shows that $\Gamma_{\max }$ and $\mathrm{cmc} / C_{20}$ are in a parallel fashion. This means if one increases, then others also increase, or if one decreases, then others also decrease. By doing so, a reduction in effectiveness of surface tension exists. Sometimes, we can see the large values of ratio $\mathrm{cmc} / C_{20}$ which may be due to steric barriers associated with the packing of these bulky chains into the micelle. One can observe that the value of $C_{20}$ plays a vital role in the increment or decrement of $\mathrm{cmc} / C_{20}$.

If the value of $C_{20}$ is increased more than the $\mathrm{cmc}$, then the $\mathrm{cmc} / C_{20}$ value is decreased, or vice versa. Also, from the above discussion, $\mathrm{cmc} / C_{20}$ is a measure of the tendency to form micelles relative to the tendency to adsorb at the air/ water interface [76].

There is a decrease or an increase in $\mathrm{cmc} / C_{20}$. A decrease in $\mathrm{cmc} / C_{20}$ indicates that the micellization facilitated more than adsorption, whereas the increase in $\mathrm{cmc} / C_{20}$ indicates that the adsorption facilitated more than micellization. Therefore, the ratio $\mathrm{cmc} / C_{20}$ provides information about the adsorption and micellization processes.

In our system, the decrease in $\mathrm{cmc} / C_{20}$ with the volume fraction of methanol indicates that the micellization facilitated more than adsorption (Figure S4). Generally, ionic surfactants with a single straight chain hydrophobic group in an aqueous system show low $\mathrm{cmc} / C_{20}$ ratios of 3 or less [46]. We also observed the value of $\mathrm{cmc} / C_{20}$ more than 2 and less than 4 for water and methanol-water mixtures at the investigated temperatures (Figure S4).

Variation in the curves for $C T A B$ with the volume fraction of methanol looks concave.

The interesting nature of the curve for CTAB at $323.15 \mathrm{~K}$ is that, at first in water, the value of $\mathrm{cmc} / C_{20}$ is the highest but decreases with the addition of methanol, whereas the value of $\mathrm{cmc} / C_{20}$ is the lowest for CTAB at $298.15 \mathrm{~K}$ in water, but decreases with the addition of methanol and finally becomes the highest (Figure S4).

3.14. Correlation of $\Delta G_{a d s}^{o} / \Delta G_{m}^{o}$ with the Volume Fraction of Methanol. The observed $\Delta G_{\text {ads }}^{o}$ values are higher than $\Delta G_{\min }^{o}$ values indicating that adsorption at the air-solution interface is more favorable than the formation of micelles in the bulk solution [77]. The ratio of $\Delta G_{\text {ads }}^{o} / \Delta G_{m}^{o}$ is found to be $<1.5$ (Figure S5) for CTAB in water and water-methanol mixture. The variation in the curves decreases for CTAB at $298.15 \mathrm{~K}$ and $308.15 \mathrm{~K}$ with the volume fraction of methanol and look concave, whereas the variation in the curve for CTAB at $318.15 \mathrm{~K}$ and $323.15 \mathrm{~K}$ with the volume fraction of methanol looks linear with the monotonous decrease in the addition of methanol (Figure S5). The curve for CTAB at $323.15 \mathrm{~K}$ is the highest, whereas at $298.5 \mathrm{~K}$, the curve of CTAB has been observed the lowest.

3.15. Correlation of $\mathrm{cmc} / p C_{20}$ with the Volume Fraction of Methanol. The ratio $\mathrm{cmc} / p C_{20}$ is an interesting study. Such type of work was observed by Niranjan and Upadhyay in 2010 [78].

The variation in the curves for CTAB increases sharply with the volume fraction of methanol and looks concave for 298.15 to $323.15 \mathrm{~K}$, whereas the variation in the curve for $\mathrm{CTAB}$ at $323.15 \mathrm{~K}$ with the volume fraction of methanol looks the highest and the lowest at $298.15 \mathrm{~K}$ (Figure S6). 
3.16. Correlation of $\mathrm{C}_{20}$ with the Volume Fraction of Methanol. $C_{20}$ is the concentration of surfactant in the bulk phase that produces a minimization of $20 \mathrm{mN} / \mathrm{M}$ in $\gamma$ of solvent. $C_{20}$ is not only a measure of the efficiency of adsorption of the liquid-gas interfaces but also a measure of the efficiency of $\gamma$ minimization by the surfactant [46]. Our calculation of $C_{20}$ for $\mathrm{CTAB}$ at $298.15 \mathrm{~K}$ is close to the literature [79].

The variation in the curves for CTAB sharply increases with the volume fraction of methanol (Figure S7), whereas the variation in the curve for $\mathrm{CTAB}$ at $323.15 \mathrm{~K}$ with the volume fraction of methanol looks the highest and the lowest at $298.15 \mathrm{~K}$.

3.17. Variation in $G_{\text {min }}$ with the Volume Fraction of Methanol. The free energy of the surface at equilibrium is also known as the molar Gibbs energy at $\mathrm{cmc}$ for maximum adsorption attained. This is one of the thermodynamic parameters for the evolution of synergism in mixed adsorption films at equilibrium [80] and is calculated as follows:

$$
G_{\min }=A_{\min } \gamma_{\mathrm{cmc}} N_{A},
$$

where $\gamma_{\mathrm{cmc}}$ is the surface tension at $\mathrm{cmc}$ and $N_{A}$ is Avogadro's number. We observed the lowest values of $G_{\min }$ in the CTAB-water system at $298.15 \mathrm{~K}$, whereas the highest value in the 0.4 volume fraction of methanol is at $323.15 \mathrm{~K}$. The observed lower values of $G_{\min }$ ascertain the thermodynamic stability [80].

The variation in the curves for CTAB with the volume fraction of methanol up to 0.2 looks as monotonous increment, whereas the curves increase sharply with the addition of more methanol (Figure S8).

The error limits of $\mathrm{cmc}, \Gamma_{\max }, A_{\text {min }}, \pi_{\mathrm{cmc}}, P$, and $\Delta G_{\text {ads }}^{\mathrm{o}}$ are within $\pm 3, \pm 5, \pm 4, \pm 3, \pm 4$, and $\pm 6 \%$, respectively.

The surface properties of CTAB in water and methanolwater mixed solvent media at $298.15 \mathrm{~K}$ were matched with the literature [21]. Let us take the equation that has the degree of dissociation $(\alpha)$, critical micelle concentration (cmc), area occupied by per surfactant molecule $\left(A_{\min }\right)$, and surface pressure $\left(\pi_{\mathrm{cmc}}\right)$ [47]:

$$
\Delta G_{\mathrm{ads}}^{\mathrm{o}}=2.303(2-\alpha) R T \log \mathrm{cmc}-0.6023 \cdot \pi_{\mathrm{cmc}} A_{\mathrm{min}},
$$

where $\alpha=0.21$ from our early studies of CTAB in water at $298.15 \mathrm{~K}$ by the conductivity technique [10] and the rest values of $A_{\text {min }}$ and $\pi_{\mathrm{cmc}}$ were taken from Table 1; then, the value of $\Delta G_{\text {ads }}^{\mathrm{o}}$ becomes $-30.837 \mathrm{~kJ} \cdot \mathrm{mol}^{-1}$. The experimental observed the standard Gibbs energy of adsorption for CTAB at 298.15 is $-63.4 \mathrm{~kJ} \cdot \mathrm{mol}^{-1}$ (Table 1 ), which is different from literature to literature: $\quad-59.62 \mathrm{~kJ} \cdot \mathrm{mol}^{-1} \quad$ [47] and $-42.01 \mathrm{kJmol}^{-1}[51]$.

\section{Conclusion}

The surface properties and micellization of CTAB in water and the presence of different volume fractions of methanolwater were investigated by surface tension measurements. The $\mathrm{cmc}$ increases with an increase in the volume fraction of methanol and with an increase in temperature. The presence of methanol shows significant changes in surface properties such as maximum surface excess concentration $\left(\Gamma_{\max }\right)$, area occupied by per surfactant molecule $\left(A_{\min }\right)$, and surface pressure $\left(\pi_{\mathrm{cmc}}\right)$. A decrease in $\Gamma_{\max }$ with increasing volume fraction of methanol-water indicates that the air/solvent interface is less populated by amphiphiles, which subsequently increase the values of $A_{\text {min }}$ and decreases the values of $\pi_{\mathrm{cmc}}$. The effect of methanol on the values of $p C_{20}$ suggests that the efficiency of adsorption decreases with an increase in the volume fraction of methanol-water to such an extent that, at 0.4 volume fraction of methanol-water, $p C_{20}$ is almost undetectable.

In short, it was discovered that, with the addition of methanol, the hydrophobicity of the medium gradually decreases, the soft interaction acting in the self-assembly of amphiphiles such as hydrogen bonding, hydrophobic effects, electrostatic interaction, and van der Waals forces, which are balancing forces required for the formation of a micelle is imbalanced, and micelles start disorganizing.

\section{Data Availability}

The data used to support the findings of this study will be available upon request to the corresponding author.

\section{Conflicts of Interest}

The authors declare that there are no conflicts of interest regarding the publication of this paper.

\section{Acknowledgments}

The authors acknowledge the University Grants Commission of Nepal and TWAS, Italy, for funding this research.

\section{Supplementary Materials}

Figures S1-S8: variation in $\pi_{\mathrm{cmc}} A_{\text {min }}$ with $\pi_{\mathrm{cmc}}$; variation in $A_{\min }$ with $1 / \pi_{\mathrm{cmc}}$; and variation in $\Gamma / \Gamma_{\max }, \mathrm{cmc} / C_{20}$, $\Delta G_{\text {ads }}^{\mathrm{o}} / \Delta G_{m}^{o}, \mathrm{cmc} / p C_{20}, C_{20}$, and $G_{\text {min }}$ with the volume fraction of methanol. (Supplementary Materials)

\section{References}

[1] N. Dubey, "CTAB aggregation in solutions of higher alcohols: thermodynamic and spectroscopic studies," Journal of Molecular Liquids, vol. 184, pp. 60-67, 2013.

[2] D. Lombardo, M. A. Kiselev, S. Magazù, and P. Calandra, "Amphiphiles self-assembly: basic concepts and future perspectives of supramolecular approaches," Advances in Condensed Matter Physics, vol. 2015, Article ID 151683, 22 pages, 2015.

[3] K. Manna and A. K. Panda, "Physicochemical studies on the interfacial and micellization behavior of CTAB in aqueous polyethylene glycol media," Journal of Surfactants and Detergents, vol. 14, no. 4, pp. 563-576, 2011.

[4] C. C. Ruiz, J. A. Molina-Bolívar, J. Aguiar, G. MacIsaac, S. Moroze, and R. Palepu, "Thermodynamic and structural studies of triton X-100 micelles in ethylene Glycol-Water mixed solvents," Langmuir, vol. 17, no. 22, pp. 6831-6840, 2001. 
[5] N. Nazir, M. S. Ahanger, and A. Akbar, "Micellization of cationic surfactant cetyltrimethylammonium bromide in mixed water-alcohol media," Journal of Dispersion Science and Technology, vol. 30, no. 1, pp. 51-55, 2009.

[6] J. Dey, D. Ray, S. Kumar et al., "Effect of acetonitrile-water mixtures on aggregation and counterion binding behavior of sodium dioctylsulphosuccinate micelles," Journal of Molecular Liquids, vol. 216, pp. 450-454, 2016.

[7] I. A. Khan, R. Mohammad, M. S. Alam, and K. ud-Din, "Surface properties and mixed micellization of cationic gemini surfactants with ethyleneamines," Journal of Chemical \& Engineering Data, vol. 55, no. 1, pp. 370-380, 2010.

[8] M. S. Alam, V. Nareshkumar, N. Vijayakumar, K. Madhavan, and A. B. Mandal, "Effect of additives on the cloud point of mixed surfactant (non-ionic Triton X-114 + cationic gemini 16-6-16) solutions," Journal of Molecular Liquids, vol. 194, pp. 206-211, 2014.

[9] M. S. Alam, A. M. Siddiq, R. Ragupathy, and A. B. Mandal, "Micellization and mixed micellization of cationic gemini (dimeric) surfactants and cationic conventional (monomeric) surfactants: conductometric, dye solubilization, and surface tension studies," Journal of Dispersion Science and Technology, vol. 38, no. 2, pp. 280-287, 2016.

[10] M. S. Alam, A. M. Siddiq, D. Natarajan, M. S. Kiran, and G. Baskar, "Physicochemical properties and bioactivity studies of synthesized counterion coupled (COCO) gemini surfactant, 1,6-bis(N,N-hexadecyldimethylammonium) adipate," Journal of Molecular Liquids, vol. 273, pp. 16-26, 2019.

[11] J. Huang and Z. H. Ren, "Micellization of binary mixture of amino sulfonate amphoteric surfactant with octylphenol polyoxyethylene ether (10) in water/ $\mathrm{NaCl}$ solution: effect of isopropanol," Journal of the Iranian Chemical Society, vol. 16, no. 6, pp. 1345-1353, 2019.

[12] Z. H. Ren, J. Huang, Y. C. Zheng, L. Lai, and L. L. Hu, "Interaction and micellar behavior of binary mixture of amino sulfonate amphoteric surfactant with octadecyltrimethylammonium bromide in aqueous solutions of $\mathrm{NaCl}$," Journal of Chemical \& Engineering Data, vol. 62, no. 6, pp. 1782-1787, 2017.

[13] Z. H. Ren, J. Huang, Y. Luo et al., "Effect of isopropanol on the micellization of binary mixtures containing amino sulfonate amphoteric surfactant in aqueous solution: mixing with octadecyltrimethyl ammonium bromide," Colloids and Surfaces A: Physicochemical and Engineering Aspects, vol. 504, pp. 131-138, 2016.

[14] I. A. Khan, R. Mohammad, M. S. Alam, and Kabir-ud-Din, "Mixed micellization of cationic gemini surfactants with primary linear alkylamines," Journal of Surfactants and Detergents, vol. 13, no. 2, pp. 179-188, 2009.

[15] M. S. Alam, N. Kamely, A. M. Siddiq et al., "Effect of dextrose and temperature on the micellization of cationic gemini surfactant (16-6-16)," Journal of Dispersion Science and Technology, vol. 36, no. 7, pp. 1029-1035, 2014.

[16] M. Sayem Alam and A. B. Mandal, "The clouding phenomena of mixed surfactant (non-ionic Triton X-114 cationic gemini 16-5-16) solutions: influence of inorganic and organic additives on the cloud point," Journal of Molecular Liquids, vol. 212, pp. 237-244, 2015.

[17] M. S. Alam and A. M. Siddiq, "Density, dynamic viscosity, and kinematic viscosity studies of aqueous solution of a cationic gemini surfactant, hexanediyl-1,6-bis(dimethylcetylammonium bromide (16-6-16): influence of electrolytes and temperature," Journal of Molecular Liquids, vol. 242, pp. 1075-1084, 2017.
[18] A. Bhattarai, S. K. Chatterjee, T. K. Deo, and T. P. Niraula, "Effects of concentration, temperature, and solvent composition on the partial molar volumes of sodium lauryl sulfate in methanol (1) + water (2) mixed solvent media," Journal of Chemical \& Engineering Data, vol. 56, no. 8, pp. 3400-3405, 2011.

[19] A. Bhattarai, "Studies of the micellization of cationic-anionic surfactant systems in water and methanol-water mixed solvents," Journal of Solution Chemistry, vol. 44, no. 10, pp. 2090-2105, 2015.

[20] S. K. Shah, S. K. Chatterjee, and A. Bhattarai, "The effect of methanol on the micellar properties of dodecyltrimethylammonium bromide (DTAB) in aqueous medium at different temperatures," Journal of Surfactants and Detergents, vol. 19, no. 1, pp. 201-207, 2015.

[21] S. K. Shah, S. K. Chatterjee, and A. Bhattarai, "Micellization of cationic surfactants in alcohol-water mixed solvent media," Journal of Molecular Liquids, vol. 222, pp. 906-914, 2016.

[22] D. M. Li, J. Huang, Z. H. Ren et al., "Interfacial properties and micellization of octadecyltrimethylammonium bromide in aqueous solution containing short chain alcohol and effect of chain length of alcohol," Journal of Dispersion Science and Technology, vol. 41, no. 6, pp. 856-862, 2020.

[23] I. Mukherjee, S. P. Moulik, and A. K. Rakshit, “Tensiometric determination of Gibbs surface excess and micelle point: a critical revisit," Journal of Colloid and Interface Science, vol. 394, pp. 329-336, 2013.

[24] A. Hugerth and L.-O. Sundelöf, "Effect of polyelectrolyte counterion specificity on dextran Sulfate-Amphiphile interaction in water and aqueous/organic solvent mixtures," Langmuir, vol. 16, no. 11, pp. 4940-4945, 2000.

[25] B. Mandal, S. Ghosh, and S. P. Moulik, "Interaction between a bio-tolerable amino-acid based amphiphile (N-dodecanoylsarcosinate, SDDS) and modified cationic polymers, hydroxyethylcelluloses (JR 400, and LM 200) in isopropanolwater medium," Colloids and Surfaces A: Physicochemical and Engineering Aspects, vol. 566, pp. 156-165, 2019.

[26] G. Kumar, M. S. Chauhan, A. Kumar, S. Chauhan, and R. Kumar, "A study on solution behavior of sodiumdodecyl sulphated and cetylteimethylammonium bromide in water-alcohol mixed media," Der Chemica Sinica, vol. 3, pp. 628-635, 2012.

[27] M. Bielawska, A. Chodzińska, B. Jańczuk, and A. Zdziennicka, "Determination of CTAB CMC in mixed water + short-chain alcohol solvent by surface tension, conductivity, density and viscosity measurements," Colloids and Surfaces A: Physicochemical and Engineering Aspects, vol. 424, pp. 81-88, 2013.

[28] A. Zdziennicka and B. Jańczuk, "Behavior of cationic surfactants and short chain alcohols in mixed surface layers at water-air and polymer-water interfaces with regard to polymer wettability. I. Adsorption at water-air interface," Journal of Colloid and Interface Science, vol. 349, no. 1, pp. 374-383, 2010.

[29] Z. H. Ren, J. Huang, Y. C. Zheng et al., "Mixed micellization of binary mixture of amino sulfonate amphoteric surfactant with octadecyltrimethyl ammonium bromide in water/isopropanol solution: comparison with that in aqueous solution," Journal of Dispersion Science and Technology, vol. 40, no. 9, pp. 1353-1359, 2018.

[30] S. K. Shah, T. P. Niraula, A. Bhattarai, and S. K. Chattarjee, "A comparative study of critical micelle concentration (CMC) and free energy of micellization of cationic surfactant and anionic surfactant in different composition of methanol-water mixed solvent media by conductometric method at $308.15 \mathrm{~K}$ ) 
of cationic surfactant (dodecyltrimethylammonium bromide, DTAB) and anionic surfactant (sodiumdodecyl sulphate, SDS) in different composition of methanol/water mixed solvent media by conductometric method at $308.15 \mathrm{~K}$," Bibechana, vol. 8, pp. 37-45, 2012.

[31] S. Z. Mikhail and W. R. Kimel, "Densities and viscosities of methanol-water mixtures," Journal of Chemical \& Engineering Data, vol. 6, no. 4, pp. 533-537, 1961.

[32] M. L. Moyá, A. Rodríguez, M. del Mar Graciani, and G. Fernández, "Role of the solvophobic effect on micellization," Journal of Colloid and Interface Science, vol. 316, no. 2, pp. 787-795, 2007.

[33] J. E. Lind Jr., J. J. Zwolenik, and R. M. Fuoss, "Calibration of conductance cells at $25^{\circ}$ with aqueous solutions of potassium Chloride1," Journal of the American Chemical Society, vol. 81, no. 7, pp. 1557-1559, 1959.

[34] A. Pan, B. Naskar, G. K. S. Prameela et al., "Amphiphile behavior in mixed solvent media I: self-aggregation and ion association of sodium dodecylsulfate in 1,4-dioxane-water and methanol-water media," Langmuir, vol. 28, no. 39, pp. 13830-13843, 2012.

[35] C. G. Bell, C. J. W. Breward, P. D. Howell, J. Penfold, and R. K. Thomas, "Macroscopic modeling of the surface tension of polymer-surfactant systems," Langmuir, vol. 23, no. 11, pp. 6042-6052, 2007.

[36] T. Chakraborty, I. Chakraborty, and S. Ghosh, "Sodium Carboxymethylcellulose-CTAB interaction: a detailed thermodynamic study of Polymer-Surfactant interaction with opposite charges," Langmuir, vol. 22, no. 24, pp. 9905-9913, 2006.

[37] S. K. Shah, "Studies on the solution properties of cationic surfactants in alcohol-water mixed solvent media," 2016, http://107.170.122.150:8080/xmlui/handle/123456789/551.

[38] S. Chakraborty, A. Chakraborty, and S. K. Saha, "Tuning of physico-chemical characteristics of charged micelles by controlling head group interactions via hydrophobically and sterically modified counter ions," RSC Advances, vol. 4, no. 61, pp. 32579-32587, 2014.

[39] K. M. Sachin, A. Chandra, and M. Singh, "Nanodispersion of flavonoids in aqueous DMSO-BSA catalysed by cationic surfactants of variable alkyl chain at $T=298.15$ to $308.15 \mathrm{~K}$," Journal of Molecular Liquids, vol. 246, pp. 379-395, 2017.

[40] L. Qin and X.-H. Wang, "Surface adsorption and thermodynamic properties of mixed system of ionic liquid surfactants with cetyltrimethyl ammonium bromide," RSC Advances, vol. 7, no. 81, pp. 51426-51435, 2017.

[41] J. N. Okorondu, L. C. Osuji, and S. E. Ofodile, "Use of biodegradation ratios in monitoring trend of biostimulated biodegradation in crude oil polluted soils," International Journal of Scientific \& Technology Research, vol. 5, pp. 179184, 2016.

[42] C. Tanford, The Hydrophobic Effect-Formation of Micelles and Biological Membranes, Wiley, New Work, NY, USA, 2nd edition, 1980.

[43] A. Bhattarai, K. Pathak, and B. Dev, "Cationic and anionic surfactants interaction in water and methanol-water mixed solvent media," Journal of Molecular Liquids, vol. 229, pp. 153-160, 2017.

[44] T. Mukhim and K. Ismail, "Aggregation, counter ion binding and adsorption behaviors of cetylpyridinium chloride in water/glycerol media at $25^{\circ} \mathrm{C}$," Journal of Surfactants and Detergents, vol. 15, no. 1, pp. 47-51, 2011.

[45] D. Das and K. Ismail, "Aggregation and adsorption properties of sodium dodecyl sulfate in water-acetamide mixtures,"
Journal of Colloid and Interface Science, vol. 327, no. 1, pp. 198-203, 2008.

[46] M. J. Rosen, Surfactants and Interfacial Phenomena, Surfactant Research Institute Brooklyn College, The City University of New York, New York, NY, USA, 2004.

[47] C. Zhang, T. Geng, Y. Jiang, L. Zhao, H. Ju, and Y. Wang, "Impact of $\mathrm{NaCl}$ concentration on equilibrium and dynamic surface adsorption of cationic surfactants in aqueous solution," Journal of Molecular Liquids, vol. 238, pp. 423-429, 2017.

[48] P. S. Niranjan, R. Shukla, and S. K. Upadhyay, "Interactions of polyacrylamide with cationic surfactants: thermodynamic and surface parameters," Journal of Surfactants and Detergents, vol. 15, no. 1, pp. 53-57, 2011.

[49] S. Javadian, V. Ruhi, A. Asadzadeh Shahir, A. Heydari, and J. Akbari, "Imidazolium-based ionic liquids as modulators of physicochemical properties and nanostructures of CTAB in aqueous solution: the effect of alkyl chain length, hydrogen bonding capacity, and anion type," Industrial \& Engineering Chemistry Research, vol. 52, no. 45, pp. 15838-15846, 2013.

[50] J. Jin, X. Li, J. Geng, and D. Jing, "Insights into the complex interaction between hydrophilic nanoparticles and ionic surfactants at the liquid/air interface," Physical Chemistry Chemical Physics, vol. 20, no. 22, pp. 15223-15235, 2018.

[51] P. K. Sansanwal, "Effect of co-solutes on the physico-chemical properties of surfactant solutions," Journal of Scientific and Industrial Research, vol. 65, pp. 57-64, 2006.

[52] M. J. Rosen and S. Aronson, "Standard free energies of adsorption of surfactants at the aqueous solution/air interface from surface tension data in the vicinity of the critical micelle concentration," Colloids and Surfaces, vol. 3, no. 3, pp. 201-208, 1981.

[53] A. Zdziennicka, K. Szymczyk, J. Krawczyk, and B. Jańczuk, "Activity and thermodynamic parameters of some surfactants adsorption at the water-air interface," Fluid Phase Equilibria, vol. 318, pp. 25-33, 2012.

[54] D. Liu, J. Ma, H. Cheng, and Z. Zhao, "Solubilization behavior of mixed reverse micelles: effect of surfactant component, electrolyte concentration and solvent," Colloids and Surfaces A: Physicochemical and Engineering Aspects, vol. 143, no. 59-68, 1998.

[55] S. Das, S. Mondal, and S. Ghosh, "Physicochemical studies on the micellization of cationic, anionic, and nonionic surfactants in water-polar organic solvent mixtures," Journal of Chemical \& Engineering Data, vol. 58, no. 9, pp. 2586-2595, 2013.

[56] S. S. Borse, T. J. Patil, and M. S. Borse, "Effect of tuned head polarity of cetyl trimethyl ammonium bromide on their physicochemical properties," Tenside Surfactants Detergents, vol. 51, no. 3, pp. 267-273, 2014.

[57] Y. Moroi, Micelles: Theoretical and Applied Aspects, Springer Science \& Business Media, Berlin, Germany, 1992.

[58] M. Almgren, P. Hansson, E. Mukhtar, and J. Van Stam, "Aggregation of alkyltrimethylammonium surfactants in aqueous poly(styrenesulfonate) solutions," Langmuir, vol. 8, no. 10, pp. 2405-2412, 1992.

[59] E. D. Goddard and K. P. Ananthapadmanabhan, Interaction of Surfactants with Polymer and Protein, CRC Press, Taylor \& Francis Group, Boca Raton, FL, USA, 2017.

[60] G. S. Hartley, Aqueous Solutions of Paraffin Chain Salts, Hermann, Paris, France, 1936.

[61] R. Zana, "Surfactant solutions," Surfactant Science Series, Vol. 22, Marcel Dekker, New York, NY, USA, 1987.

[62] P. Debye and E. W. Anacker, "Micelle shape from dissymmetry measurements," The Journal of Physical Chemistry, vol. 55, no. 5, pp. 644-655, 1951. 
[63] R. J. Vetter, "Micelle structure in aqueous solutions of colloidal electrolytes," The Journal of Physical and Colloid Chemistry, vol. 51, no. 1, pp. 262-277, 1947.

[64] J. B. Peri, "The state of dispersion of detergent additives in lubricating oil and other hydrocarbons," Journal of the American Oil Chemists' Society, vol. 35, no. 3, pp. 110-117, 1958.

[65] C. W. Dwiggins Jr., R. J. Bolen, and H. N. Dunning, "Ultracentrifugal determination of the micellar character of nonionic detergent Solutions," The Journal of Physical Chemistry, vol. 64, no. 9, pp. 1175-1178, 1960.

[66] D. Bendedouch, S. H. Chen, and W. C. Koehler, "Determination of interparticle structure factors in ionic micellar solutions by small angle neutron scattering," The Journal of Physical Chemistry, vol. 87, no. 14, pp. 2621-2628, 1983.

[67] J. B. Hayter and J. Penfold, "Self-consistent structural and dynamic study of concentrated micelle solutions," Journal of the Chemical Society, Faraday Transactions 1: Physical Chemistry in Condensed Phases, vol. 77, p. pp1851, 1981.

[68] P. G. Nilsson, H. Wennerstroem, and B. Lindman, "Structure of micellar solutions of nonionic surfactants. Nuclear magnetic resonance self-diffusion and proton relaxation studies of poly(ethylene oxide) alkyl ethers," The Journal of Physical Chemistry, vol. 87, no. 8, pp. 1377-1385, 1983.

[69] P. G. Nilsson and B. Lindman, "Water self-diffusion in nonionic surfactant solutions. Hydration and obstruction effects," The Journal of Physical Chemistry, vol. 87, no. 23, pp. 4756-4761, 1983.

[70] B. Lindman, "Structural aspects of surfactant micellar systems," in Surfactants, T. F. Tadros, Ed., p. 83, Academic Press, New York, NY, USA, 1984.

[71] M. N. Wadekar, J. Boekhoven, W. F. Jager, G. J. M. Koper, and S. J. Picken, "Micellization behavior of aromatic moiety bearing hybrid fluorocarbon sulfonate surfactants," Langmuir, vol. 28, no. 7, pp. 3397-3402, 2012.

[72] J. da Silva, R. Dias, G. da Hora, T. Soares, and M. Meneghetti, "Molecular dynamics simulations of cetyltrimethylammonium bromide (CTAB) micelles and their interactions with a gold surface in aqueous solution," Journal of the Brazilian Chemical Society, vol. 29, no. 1, pp. 191-199, 2018.

[73] A. Malliaris, J. Le Moigne, J. Sturm, and R. Zana, “Temperature dependence of the micelle aggregation number and rate of intramicellar excimer formation in aqueous surfactant solutions," The Journal of Physical Chemistry, vol. 89, no. 12, pp. 2709-2713, 1985.

[74] E. D. Goddard and R. B. Hannan, "Cationic polymer/anionic surfactant interactions," Journal of Colloid and Interface Science, vol. 55, no. 1, pp. 73-79, 1976.

[75] E. D. Goddard, O. Kao, and H. C. Kung, "Counterion effects in charged monolayers," Journal of Colloid and Interface Science, vol. 27, no. 4, pp. 616-624, 1968.

[76] G. Sugihara, S. Nagadome, S.-W. Oh, and J.-S. Ko, "A review of recent studies on aqueous binary mixed surfactant systems," Journal of Oleo Science, vol. 57, no. 2, pp. 61-92, 2008.

[77] R. Patel, A. B. Khan, N. Dohare, M. Maroof Ali, and H. K. Rajor, "Mixed micellization and interfacial properties of ionic liquid-type imidazolium gemini surfactant with amphiphilic drug amitriptyline hydrochloride and its thermodynamics," Journal of Surfactants and Detergents, vol. 18, no. 5, pp. 719-728, 2015.

[78] P. S. Niranjan and S. K. Upadhyay, "Interaction of polyacrylamide with conventional anionic and gemini anionic surfactants," Journal of Dispersion Science and Technology, vol. 32, no. 1, pp. 109-113, 2010.

[79] T. S. Banipal and A. K. Sood, "Mixed micellar and interfacial interactions of a triblock polymer (EO37PO56EO37) with a series of monomeric and dimeric surfactants," Journal of Surfactants and Detergents, vol. 16, no. 6, pp. 881-891, 2013.

[80] P. Parekh, D. Varade, J. Parikh, and P. Bahadur, "Anioniccationic mixed surfactant systems: micellar interaction of sodium dodecyl trioxyethylene sulfate with cationic gemini surfactants," Colloids and Surfaces A: Physicochemical and Engineering Aspects, vol. 385, no. 1-3, pp. 111-120, 2011. 